\title{
Experience-Induced Neurogenesis in the Senescent Dentate Gyrus
}

\author{
Gerd Kempermann, ${ }^{1}$ H. Georg Kuhn, ${ }^{1,2}$ and Fred H. Gage ${ }^{1}$ \\ ${ }^{1}$ The Salk Institute for Biological Studies, Laboratory of Genetics, La Jolla, California 92037, and 2Department of \\ Neurology, University of Regensburg, D-93053 Regensburg, Germany
}

\begin{abstract}
We demonstrate here that under physiological conditions neurogenesis continues to occur in the dentate gyrus of senescent mice and can be stimulated by living in an enriched environment. Neurogenesis was investigated by confocal microscopy of three-channel immunofluorescent staining for the proliferation marker bromodeoxyuridine (BrdU) and neuronal and glial markers. Quantification was performed with unbiased stereological counting techniques. Neurogenesis decreased with increasing age. Stimulation of adult and aged mice by switching from standard housing to an enriched environment with opportunities for social interaction, exploration, and physical activity for $68 \mathrm{~d}$ resulted in an increased survival of labeled cells. Phenotypic analysis revealed that, in enriched living animals, relatively more cells differentiated into neurons, resulting in a
\end{abstract}

threefold net increase of BrdU-labeled neurons in 20-month-old mice (105 vs 32 cells) and a more than twofold increase in 8 -month-old mice (684 vs 285 cells) compared with littermates living under standard laboratory conditions. Corresponding absolute numbers of BrdU-positive astrocytes and BrdU-positive cells that did not show colabeling for neuronal or glial markers were not influenced. The effect on the relative distribution of phenotypes can be interpreted as a survival-promoting effect that is selective for neurons. Proliferation of progenitor cells appeared unaffected by environmental stimulation.

Key words: aging; brain; mouse; hippocampus; neurogenesis; stem cell; progenitor cell; enriched environment; plasticity; stereology
New neurons are continuously born in the dentate gyrus of the hippocampal formation in adult mammals. At the age of 2 months, the dentate gyrus of adult C57BL/6 mice produces an average of approximately one new granule cell neuron per 1700 existing granule cells per day (Kempermann et al., 1997a). The functional significance of these new cells, however, remains elusive.

The aging hippocampal formation is affected by a number of structural and functional changes, including the loss of neurons in CA regions and the hilus (West et al., 1994), reduced synaptic densities (Saito et al., 1994), and reduced expression of growth factor and steroid receptors (Bhatnagar et al., 1997). Neurogenesis in the dentate gyrus occurs throughout the life of a rat (Altman and Das, 1965; Kaplan and Bell, 1984; Cameron et al., 1993; Kuhn et al., 1996) but decreases with increasing age (Kuhn et al., 1996). The extent to which the remaining neurogenesis in the dentate gyrus is regulated and has functional consequences is still unknown.

We have used the experimental paradigm of an enriched environment (Rosenzweig et al., 1962; Rosenzweig and Bennett, 1996) to demonstrate experience-induced plasticity of neuronal numbers in the dentate gyrus (Kempermann et al., 1997b). This finding placed adult neurogenesis in the dentate gyrus in a func-

\footnotetext{
Received Dec. 3, 1997; revised Feb. 10, 1998; accepted Feb. 17, 1998.

This study was supported by grants from National Institute of Neurological Diseases and Stroke, National Institute on Aging, American Paralysis Association, and International Spinal Research Trust. G.K. was supported by Deutsche Forschungsgemeinschaft, and H.G.K. was supported by the Hereditary Disease Foundation. We thank D. A. Peterson for help with stereological questions and T. D. Palmer, P. J. Horner, J. Winkler, and M. L. Gage for comments on this manuscript. We also thank A. Smith, T. Slimp, K. Suter, and their colleagues from the Animal Research Facilities of the Salk Institute for their support of these experiments.

Correspondence should be addressed to Dr. Fred H. Gage, The Salk Institute for Biological Studies, Laboratory of Genetics, 10010 North Torrey Pines Road, La Jolla, CA 92037.

Copyright (ㄷ) 1998 Society for Neuroscience $\quad 0270-6474 / 98 / 183206-07 \$ 05.00 / 0$
}

tional context and raised the question of whether use-dependent neuroplasticity is possible in the old brain.

In the present study, we examined the effects of living in an enriched environment on neurogenesis in the dentate gyrus of C57BL/6 mice at the ages of 6 and 18 months. The four groups of mice in the study are referred to as Ctr-6, 6-month-old control; Ctr-18, 18-month-old control; Enr-6, 6-month-old enriched; and Enr-18, 18-month-old enriched. At the end of the experiment the animals were 8 and 20 months old, respectively. These ages represent midlife and senescence, considering that the average life span of a female C57BL/6 mouse is 26 months (Kunstyr and Leuenberger, 1975) and the mouse menopause is at $\sim 13$ months of age (Silver, 1995). Before the experimental period, which lasted 40 or $68 \mathrm{~d}$, all animals were housed under standard laboratory conditions. Neurogenesis was examined by (1) incorporation of bromodeoxyuridine (BrdU) in the DNA of proliferating cells and immunohistochemical detection of BrdU, (2) immunofluorescent triple-labeling for BrdU and neuronal and glial markers and confocal laser microscopy to examine the phenotypes of surviving BrdU-positive cells, and (3) unbiased stereological counting techniques to obtain absolute cell numbers. In addition, the animals were tested on a water maze task to examine spatial learning as a measure of hippocampal function.

\section{MATERIALS AND METHODS}

Housing conditions. Fifty female C57BL/6 mice, half 6 months old and the other half 18 months old, were obtained from the aging colony at the National Institute of Aging. Twelve animals per age group were housed in four standard cages; 13 per age group were placed in an enriched environment. Enriched living conditions are described in Figure 1 and did not differ from the set-up in our previous study (Kempermann et al., 1997b). There were separate cages with enriched environments for each age group. The time course of the experiments is displayed in Figure 2. Animals lived in their respective experimental conditions for $40 \mathrm{~d}$. During the last $12 \mathrm{~d}$ they received BrdU injections (see below). On day 41 five animals from each group were killed with an overdose of anes- 

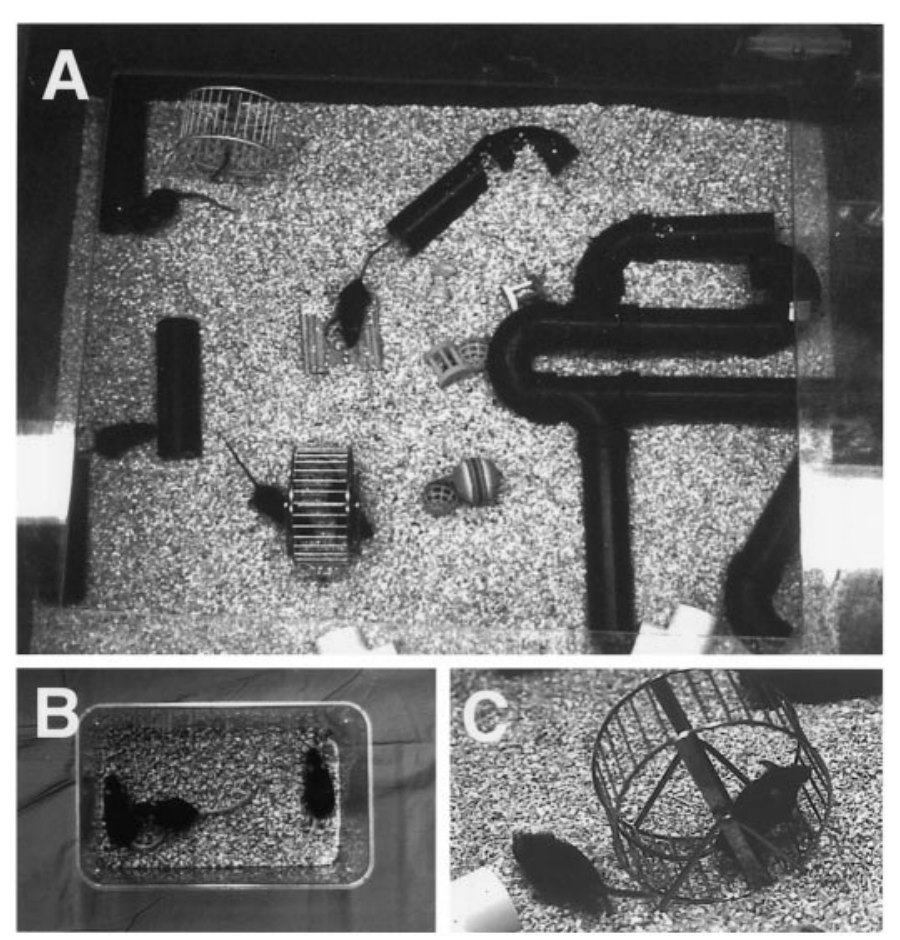

Figure 1. $A$, One of the cages for an enriched environment; $B$, One standard cage at the same scale (the trays for food and water are removed). Enrichment consisted of social interaction (13 mice in the large cage vs 3 in the standard cage), stimulation of exploratory behavior with objects such as toys and a rearrangeable set of tunnels, and $C$, physical activity in a running wheel. In addition to standard food and water $a d$ libitum, enriched mice received occasional treats, including cheese, crackers, and fruits.

thetics and perfused transcardially with cold $4 \%$ paraformaldehyde in 0.1 M PBS. The remaining animals continued to live under their respective experimental conditions for $28 \mathrm{~d}$. During this time they were tested on the water maze task (see below) and perfused on day 68 .

BrdU injections. BrdU (Sigma, St. Louis, MO) was dissolved in $0.9 \%$ $\mathrm{NaCl}$ and filtered sterile at $22 \mu \mathrm{m}$. The mice received single doses of 50 $\mu \mathrm{g} / \mathrm{gm}$ of body weight at a concentration of $10 \mathrm{mg} / \mathrm{ml}$, one intraperitoneal injection per day for 12 consecutive days (Fig. 2).

Water maze testing. Seven mice from each group were tested with two trials per day over $10 \mathrm{~d}$. The platform was hidden below the surface of the opaque water. The two starting points were changed daily. Each trial lasted either until the mouse had found the platform or for a maximum of $60 \mathrm{sec}$. All mice were allowed to rest on the platform for $10 \mathrm{sec}$. Analyses of time to reach the platform (latency), length of swim path, and swim speed were recorded semiautomatically by a video tracking system (San Diego Instruments). On day 11 a probe trial with the platform removed was performed and did not show differences among the groups.

Immunohistochemistry. Immunohistochemistry for BrdU and immunofluorescent triple labeling for BrdU, NeuN, and $\mathrm{S} 100 \beta$ was performed as described previously (Kuhn et al., 1997). All staining was done on free-floating $40 \mu \mathrm{m}$ sections that were pretreated for BrdU immunohistochemistry by denaturing DNA. The antibodies used were mouse antiBrdU (Boehringer Mannheim, Indianapolis, IN), 1:400; rat anti-BrdU ascites (Accurate; for triple-labeling), 1:100; rabbit anti-S100 $\beta$ (Swant), 1:2500; and mouse anti-NeuN (kindly provided by R. J. Mullen, University of Utah, Salt Lake City, UT), 1:20. To determine the number of BrdU-positive cells, staining for BrdU with the peroxidase method was used (ABC system, with biotinylated horse anti-mouse antibodies and diaminobenzedine as chromogen; Vector Laboratories, Burlingame, CA). The fluorescent secondary antibodies used were anti-mouse FITC, anti-rat Texas Red, and anti-rabbit CY-5 (Jackson ImmunoResearch, West Grove, PA), $6 \mu \mathrm{l} / \mathrm{ml}$.

Analysis of phenotypes. Sections from animals surviving 4 weeks after the last injection of BrdU were triple-labeled as described above and analyzed by confocal microscopy (Zeiss, Bio-Rad, Richmond, CA). In total, 50 BrdU-positive cells per animal were analyzed for coexpression of BrdU and NeuN for neuronal phenotype and S100 $\beta$ for glial phenotype. To achieve this number of analyses over the rostrocaudal extension of the dentate gyrus, a 1 of 12 series of $40 \mu \mathrm{m}$ sections in Ctr- 6 and Enr- 6 and a one of six series in Ctr-18 and Enr-18 were required. The microscopic analysis yielded a ratio of BrdU-positive cells colabeling with (1) NeuN, (2) S100 $\beta$, or (3) neither NeuN nor S100 $\beta$. These ratios were multiplied with the total number of surviving BrdU-labeled cells to give an estimate of the total number of BrdU-positive neurons, BrdU-positive astrocytes, and BrdU-positive cells that acquired neither phenotype during the 4 weeks after the last BrdU injection.

Stereology. The number of granule cells was determined in every sixth section in a series of $40 \mu \mathrm{m}$ coronal sections using unbiased stereology (optical disector; Gundersen et al., 1988). A recent commentary seemed to suggest that the optical disector method has few benefits over traditional, so-called intuitive counting techniques, when these are combined with appropriate correction procedures (Guillery and Herrup, 1997). Although the disector method is not assumption-free and therefore not absolute or objective in a very strict sense, it has the following advantages: (1) the structure volume is taken into consideration; (2) the procedure can be computerized to a large degree; and (3) the results are highly reliable and as absolute or objective as technically possible (Gundersen et al., 1988; Coggeshall and Lekan, 1996). The words absolute or total are used here in a practical, not a theoretical or philosophical, sense.

Systematic samplings of unbiased counting frames of $15 \mu \mathrm{m}$ on a side with a $200 \mu \mathrm{m}$ matrix spacing were produced using a semiautomatic stereology system (StereoInvestigator, MicroBrightfield, Inc.) and a $60 \times$ SPlan apo oil objective (1.4 numerical aperture). Granule cells were stained with Hoechst 33342 (Molecular Probes, Eugene, OR; $0.5 \mathrm{mg} / \mathrm{ml}$ Tris-buffered saline for $15 \mathrm{~min})$; those that intersected the uppermost focal (exclusion) plane and those that intersected the exclusion boundaries of the unbiased sampling frame were excluded from counting. Cells that met the counting criteria through a $40 \mu \mathrm{m}$ axial distance were counted according to the optical disector principle. The granule cell layer reference volume was determined by summing the traced granule cell areas for each section multiplied by the distance between sections sampled. The mean granule cell number per disector volume was multiplied by the reference volume to estimate the total granule cell number. Because BrdU-positive cells were so rarely encountered, sampling of these cells was done exhaustively throughout the extent of the granule cell layer, modifying the optical disector procedure to exclude the top focal plane only, and omitting the exclusion and inclusion boundaries of

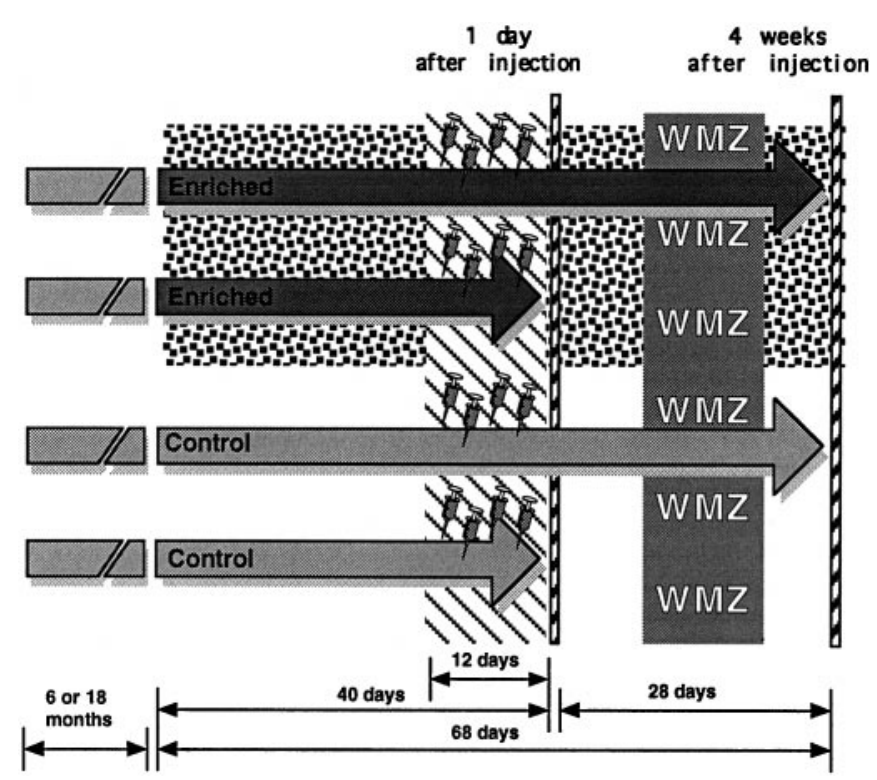

Figure 2. Experimental design applied to each of the two age groups (6 or 18 months at the beginning of the experiment). The $12 \mathrm{~d}$ period of daily BrdU injections is symbolized by syringes. WMZ, Behavioral testing on the water maze task. 
$A^{\text {No. of BrdU-positive cells (1 day/4 weeks) }}$

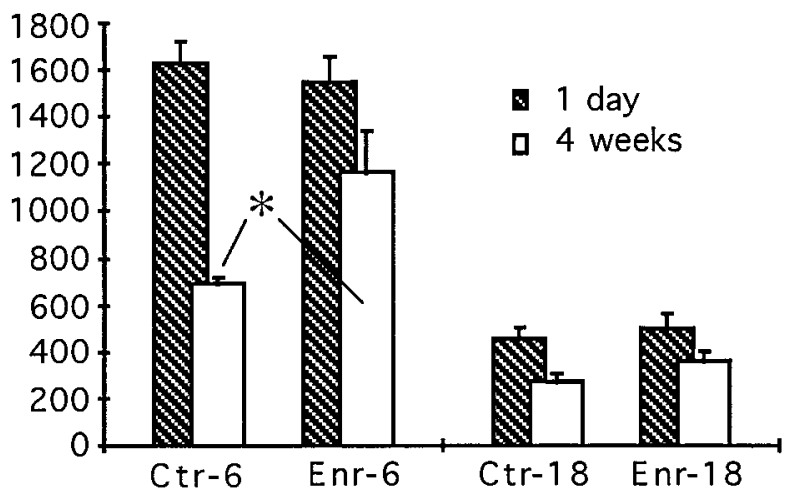

\section{$B$ Phenotypes at age 6 months}

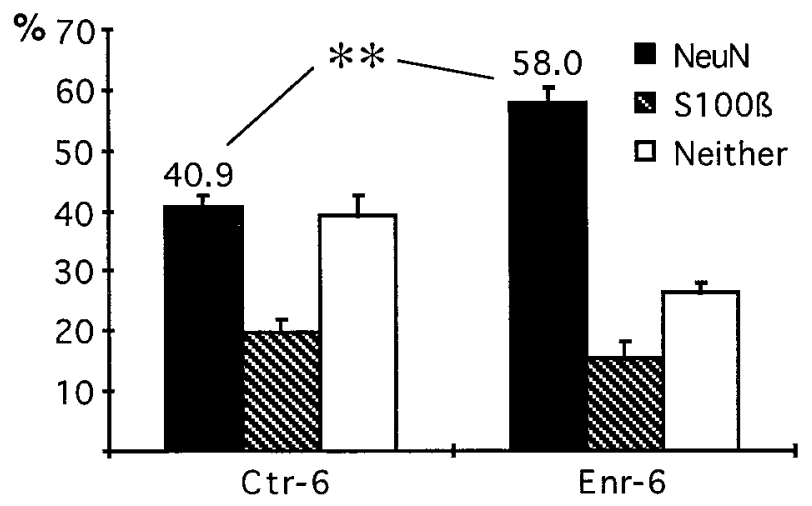

Phenotypes at age 18 months

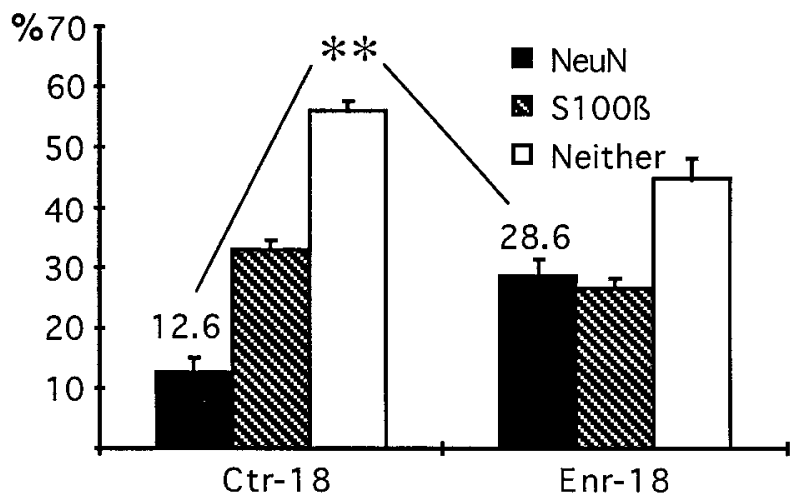

D BrdU-positive neurons per dentate
gyrus (4 weeks after injection)

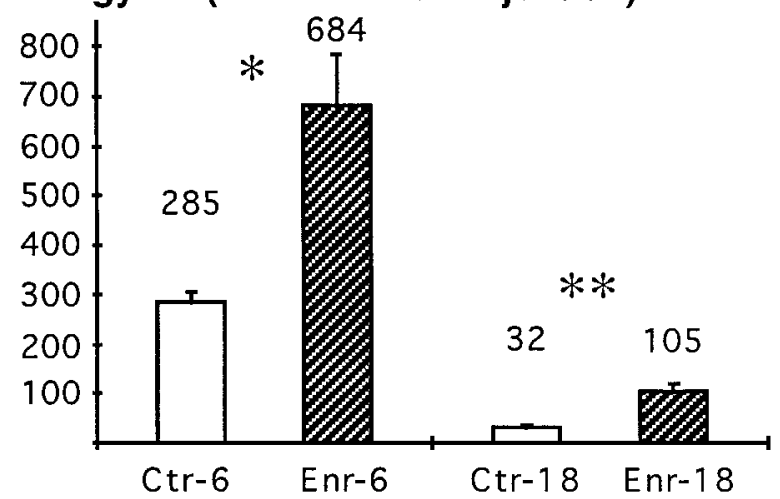

Figure 3. A, Total number of BrdU-positive cells per dentate gyrus $1 \mathrm{~d}$ after the last injection of BrdU to estimate ongoing proliferation (hatched bars) and 4 weeks later to assess survival of BrdU-positive cells (open bars). B, $C$, Percentage of the surviving BrdU-positive cells (compare with $B$ ) that differentiated into either neuronal ( filled bars) or glial (hatched bars) phenotype or showed neither differentiation (open bars). $D$, the total number of BrdU-labeled neurons generated over the $12 \mathrm{~d}$ of BrdU injections (values derived from the phenotypic ratio times the number of BrdU-positive cells at 4 weeks). Note that because the number of BrdU-positive cells is influenced by cell cycle parameters, no absolute statements about the size of the population of proliferating neuronal progenitor cells are possible. ${ }^{*} p<0.05 ;{ }^{*} p<0.005$. For details on statistical analyses see Materials and Methods.

the unbiased sample frame. The resulting number of BrdU-positive cells was then related to the granule cell layer sectional volume and multiplied by the reference volume to estimate the total number of BrdU-positive cells.

Statistical analysis. All statistical analyses were performed with Statview 4.01 for Macintosh. For all comparisons of morphological data factorial ANOVAs were performed followed by a Fisher's post hoc test, when appropriate. The level of significance was assumed to be $5 \%$. To assess the percentage of surviving BrdU-positive cells, the individual values of the numbers of BrdU-positive cells at 4 weeks after injection were divided by the mean number of BrdU-positive cells at $1 \mathrm{~d}$ after injection. Water maze data were analyzed within each age group by ANOVA with repeated measures, followed by Fisher's post hoc test. The mean values of the two trials per day were regarded as repeated measures for overall analysis over days.

\section{RESULTS}

Neurogenesis, defined as birth of new nerve cells, consists of a series of distinct developmental steps, three of which can be examined separately: proliferation, survival, and differentiation.

Proliferation of progenitor cells in the subgranular zone was addressed by BrdU labeling of dividing cells and consecutive immunohistochemical analysis with antibodies against BrdU $1 \mathrm{~d}$ after the last injection of BrdU. At this time point we did not see any significant effect of living in an enriched environment on proliferation of cells in the dentate gyrus (Fig. $3 A$ ). However, proliferation of hippocampal progenitor cells decreased with age, because senescent mice (Ctr-18) had significantly fewer BrdUpositive cells compared with mice at medium age (Ctr-6) $(p<$ 0.0001).

Survival of the progeny of dividing progenitor cells can be addressed by staining for BrdU-positive cells 4 weeks after the last injection of BrdU and comparing the number of labeled cells with the number obtained at $1 \mathrm{~d}$ after the last injection (Fig. $3 A$ ). We found that the total number of surviving BrdU-positive cells after 4 weeks significantly decreased in old age (Ctr-6 vs Ctr-18; $p=0.0081)$. Living in an enriched environment increased the number of surviving labeled cells by $68 \%$ at age 6 months $(p=$ $0.0025)$ and by $32 \%$ in 18 -month-old mice ( $p>0.05$; Fig. $3 A)$. When the number of surviving cells was expressed as a ratio of the number of cells at $1 \mathrm{~d}$ after the last injection, it was found that age alone had no significant survival-promoting effect. In $\mathrm{Ctr}-6$ on average $42.7 \pm 1.8 \%$ of the labeled cells at $1 \mathrm{~d}$ after injection were detectable 4 weeks later, whereas $60.7 \pm 7.3 \%$ were found in Ctr-18 ( $p=0.1540$; all values mean $\pm \mathrm{SEM})$.

Differentiation of the surviving BrdU-positive cells was examined 4 weeks after the last injection by determining the phenotypes into which surviving BrdU-positive cells had differentiated by means of confocal microscopy and immunofluorescent triple labeling for BrdU, neuronal marker NeuN (Mullen et al., 1992), and glial marker S100 $\beta$ (Boyes et al., 1986) (Fig. 4). In Enr-18, 

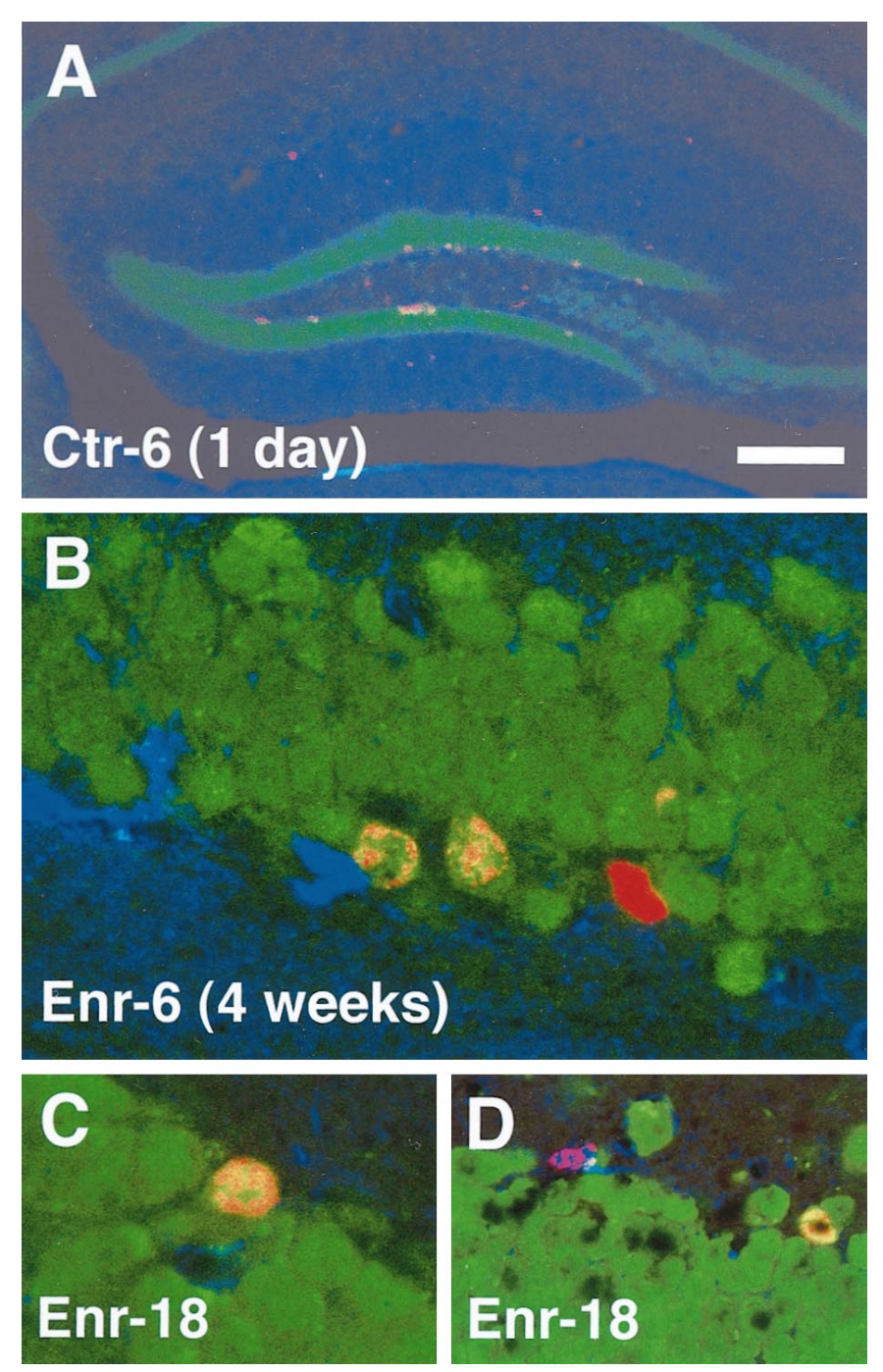

Figure 4. Confocal microscopic analysis of immunofluorescent triplelabeling of BrdU-positive cells (red) $1 \mathrm{~d}(A)$ and 4 weeks $(B-D)$ after the last injection of BrdU. $A$, Overview of the distribution of BrdU-positive cells along the border between the arrowhead-shaped granule cell layer of the dentate gyrus and the hilar area CA4 (compare with hatched columns in Fig. 3A). In addition there are some proliferating cells in the hilus itself and in the molecular layer. No qualitative difference among the four groups could be found. Four weeks after injection the phenotypes of BrdU-labeled cells were examined $(B-D)$. Markers were NeuN (green) for neurons and $\mathrm{S} 100 \beta$ (blue) for astrocytes. $B$, Two BrdU-labeled neurons (orange $=$ red + green) and one BrdU-positive cell that is neither NeuN- nor S100 $\beta$-positive in an Enr-6 animal. $C$, BrdU-labeled neuron with the typical chromatin pattern of a granule cell in an Enr-18 animal. $D$, One BrdU-labeled astrocyte $($ pink $=$ red + blue, left $)$ and one BrdUlabeled neuron (orange, right). Scale bar (in $A$ ): $A, 200 \mu \mathrm{m} ; B, C, 12 \mu \mathrm{m}$; $D, 20 \mu \mathrm{m}$.

neurons (NeuN-positive cells) accounted for $28.6 \pm 8.6 \%$ of the surviving BrdU-positive cells versus $12.6 \pm 7.3 \%$ in $\mathrm{Ctr}-18$ ( $p=$ $0.0002)$; in Enr-6 we found $58.0 \pm 6.8 \%$ neurons versus $40.8 \pm$ $4.7 \%$ in Ctr-6 $(p<0.0001$; Fig. $3 B, C)$.

Enr-18 mice generated on average a total of $105 \pm 18$ BrdUlabeled neurons per dentate gyrus versus only $32 \pm 7$ in Ctr-18 mice during the $12 \mathrm{~d}$ of BrdU injection (a threefold increase; $p=$ 0.0035) (Fig. 3D). By comparison, Enr-6 produced $684 \pm 104$ BrdU-labeled neurons, whereas Ctr-6 produced an average of $285 \pm 22$ (a twofold increase; $p=0.0109$ ). This effect was greater in absolute terms at 6 months but greater in relative terms at 18 months.

At 4 weeks after the last injection of BrdU, astrocytes (S100 $\beta$ positive cells) accounted for $19.7 \pm 3.2 \%$ of surviving BrdUpositive cells in Ctr-6, $15.5 \pm 1.7 \%$ in Enr-6, $32.9 \pm 2.0 \%$ in Ctr-18, and $26.6 \pm 1.8 \%$ in Enr-18. There was no significant difference between Ctr-6 and Enr-6 $(p=0.1864)$ and Ctr-18 and Enr-18 $(p=0.0553)$. In absolute terms, there were $133 \pm 20$ BrdU-labeled astrocytes in Ctr-6, $185 \pm 38$ in Enr-6, $92 \pm 13$ in Ctr-18, and $99 \pm 16$ in Enr-6. Again, no significant difference between Ctr-6 and Enr-6 ( $p=0.1516)$ and Ctr-18 and Enr-18 ( $p=0.8389)$ was found.

BrdU-positive cells that did not show colocalization with either NeuN of S100 $\beta$ accounted for $39.4 \pm 2.4 \%$ in Ctr-6 (absolute number, $275 \pm 23), 26.5 \pm 2.8 \%$ in Enr-6 (299 \pm 49$), 56.0 \pm 1.6 \%$ in Ctr-18 (154 \pm 20$)$, and $44.8 \pm 9.3 \%$ in Enr-18 (160 \pm 22$)$. Statistical analysis showed that the relative values were significantly different in both age groups ( $p=0.0027$ for Ctr-6 vs Enr-6; $p=0.0079$ for Ctr. 18 vs Enr-18) but not the absolute numbers ( $p=0.6099$ and $p=0.8965$, respectively).

The effects of enrichment-induced neurogenesis on total granule cell numbers were assessed by means of unbiased stereological techniques (Table 1). However, in this comparison of two cellular populations of very different size, the interindividual variance in the total granule cell number within each group surpassed the total number of neurons that were born during the $12 \mathrm{~d}$ injection period $(<4000$ in Enr-6). Thus, we did not find any differences in total granule cell numbers among the four groups; neither was there any difference with regard to the mean neuronal density in the granular layer or the volume of the granular layer.

It has been reported that old mice learn in the water maze task (Gower and Lamberty, 1993). Overall analysis of the water maze test in our study revealed significantly shorter times to reach the platform when comparing Enr-18 versus Ctr-18 ( $p=0.0422$; Fig. $5 A)$ and Enr-6 versus Ctr-6 $(p=0.0248)$. Although we found a significant increase in swim speed in both Enr-18 versus Ctr-18 $(p=0.0307$; Fig. 5B) and Enr-6 versus Ctr-6 $(p=0.0040)$, there was no difference in the lengths of swim paths in enriched animals versus controls.

\section{DISCUSSION}

Neurogenesis means birth of new neurons, but is often used in the restricted sense of cell proliferation leading to a new neuron, although broader definitions are in use (Shepherd, 1994; Caviness et al., 1995). The adult hippocampal formation contains multipotent stem cells, differentiating to both neurons and glial cells in vitro (Palmer et al., 1997). To base the definition of neurogenesis on a process that could also result in glia is ambiguous. In contrast to the precise temporospatial distribution of neurogenic events during embryonic development (Caviness et al., 1995), regions of adult neurogenesis contain cells at all developmental stages. Consequently, the use of proliferation to define neurogenesis in the adult brain is less precise than during development. Comparing data from $1 \mathrm{~d}$ with 4 weeks after BrdU injection revealed that the amount of proliferation is not indicative of the resulting net production of new neurons (Kempermann et al., 1997a). We therefore use a broader definition of neurogenesis that includes proliferation, survival, and differentiation.

\section{Neurogenesis in the senescent dentate gyrus}

Neurogenesis persists in the hippocampal formation of old mice. In all four groups, proliferating cells (Fig. $4 A$ ) were located in the 


\begin{tabular}{lcccc}
\hline Table 1. Stereological data from the hippocampus of mice & & & \\
& Ctr-6 & Enr-6 & Ctr-18 & Enr-18 \\
\hline Volume of the granule cell layer $\left(\mathrm{mm}^{3}\right)$ & $0.356 \pm 0.009$ & $0.373 \pm 0.008$ & $0.336 \pm 0.010$ & $0.365 \pm 0.013$ \\
Neuronal density & $9.91 \pm 0.37$ & $9.47 \pm 0.23$ & $9.55 \pm 0.40$ \\
Absolute number of granule cells & $3.90 \times 10^{5} \pm 0.13 \times 10^{5}$ & $3.91 \times 10^{5} \pm 0.08 \times 10^{5}$ & $3.57 \times 10^{5} \pm 0.18 \times 10^{5}$ & $3.74 \times 10^{5} \pm 0.16 \times 10^{5}$ \\
\hline
\end{tabular}

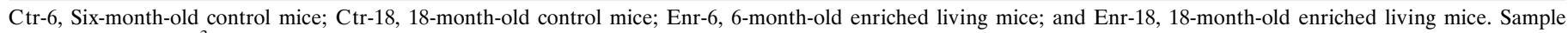
volume was $9000 \mu \mathrm{m}^{3}$. The numbers are mean \pm SEM. ANOVA revealed that no significant differences exist among the groups.
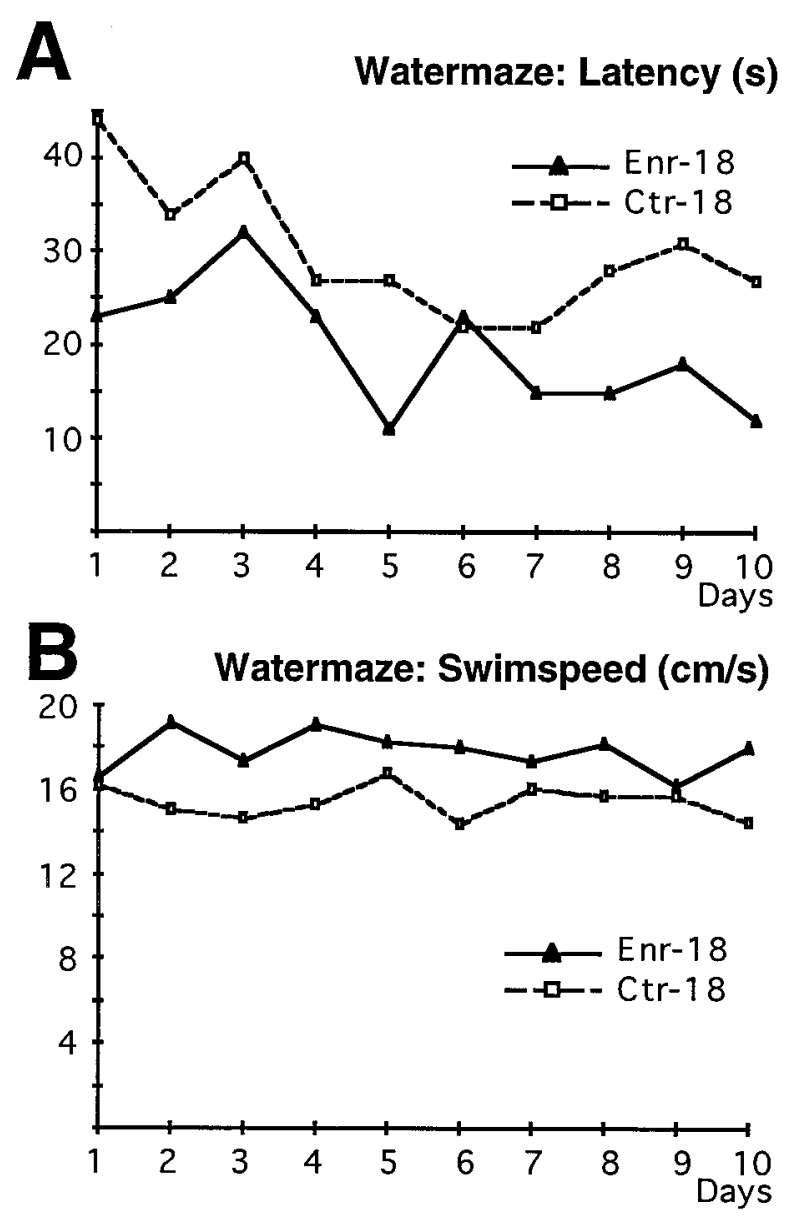

Figure 5. Data from the water maze test for 18-month-old mice. Performance curves for 6-month-old animals looked similar. Overall analysis of time to reach the platform revealed significant differences between controls and enriched living mice. The curve for swim path paralleled $A$ in appearance but in overall analysis no significant difference between the groups could be found. Although experience induced functional improvements, a causal relation to neurogenesis remains to be established.

subgranular zone, and differentiating cells migrated into the granule cell layer. BrdU-labeled cells expressed the neuronal marker NeuN 4 weeks after injection of BrdU (Fig. $4 B-D$ ).

The decrease in neurogenesis with increasing age confirmed previous results from rats (Kuhn et al., 1996). We found no significant difference in the total granule cell number between the two age groups, indicating that in old age neurogenesis ceases to produce granule cells in numbers that influence the total granule cell count at a detectable rate. Comparison with data from our previous study reveals an increase in the total granule cell number between 2 and 6 months by $\sim 40,000$ cells (Kempermann et al., 1997a). Comparable increases have been described for rats (Bayer et al., 1982).
Little is known about the role of apoptosis in the regulation of adult neurogenesis, although it is plausible that the decrease in BrdU-positive cells during 4 weeks after injection is attributable to elimination by programmed cell death. Apoptotic nuclei have been described in the subgranular zone (Bengzon et al., 1997). Quantitatively, the loss of BrdU-positive cells might reflect an overestimation of apoptosis, because BrdU itself could damage some labeled cells.

Birth dating cells by BrdU incorporation is influenced by cell cycle parameters (Cai et al., 1997). Because BrdU has a bioavailability of $\sim 2 \mathrm{hr}$ (Takahashi et al., 1992), only cells that are in S phase in this short period incorporate BrdU. A lengthened S phase will increase the probability of a cell being labeled during each injection; however, during the period of injections fewer cell cycles are likely to occur. BrdU-positive cells that divide again after BrdU is eliminated from the bloodstream will give BrdU to their daughter cells. The rationale for $12 \mathrm{BrdU}$ injections over $12 \mathrm{~d}$ was to minimize the impact of divisions after discontinuation of BrdU, to label more cells, and to compensate for dilution effects caused by continued divisions.

\section{Experience-induced regulation of neurogenesis}

The paradigm of environmental enrichment (Rosenzweig et al., 1962) has been applied to study the environmental effect on cellular proliferation in the adult brain (Altman and Das, 1964). We have recently shown that stimulating mice with an enriched environment results in increased neurogenesis in the dentate gyrus (Kempermann et al., 1997b).

Our present results indicate that experience-dependent regulation of adult neurogenesis occurs in the aging dentate gyrus. As in younger mice, survival of BrdU-labeled cells was promoted. The underlying mechanism could lie in an interaction with the apoptotic cascade.

In addition, we found that in enriched-living animals of both age groups relatively more BrdU-positive cells double labeled for neuronal marker $\mathrm{NeuN}$, an effect that was not apparent in 3-month-old mice (Kempermann et al., 1997b). This can be interpreted as a survival-promoting effect on cells that are committed to a neuronal lineage. The absolute numbers of both BrdU-labeled astrocytes and BrdU-positive cells, which did not double label for NeuN or $\mathrm{S} 100 \beta$, were not influenced by environmental stimuli-a finding that supports this hypothesis.

An alternative hypothesis that remains to be tested is that environmental stimuli could have an effect on the differentiation of progeny of the dividing progenitor cells by directly or indirectly influencing mechanisms of cell fate decisions.

The microenvironmental conditions and their experiencedependent alteration, which favor the survival of cells that have exited the cell cycle and which lead these cells toward differentiation, remain to be identified. Although gliogenesis was not significantly stimulated by experience in old animals, it remains possible that environmental influences on astrocytes contribute to the overall effects. 
That there was no difference in proliferation between controls and enriched-living mice does not allow conclusions about the size of the progenitor population. In principle, our findings on proliferation would be consistent with either a smaller proliferating population with shorter cell cycles or a larger population with longer cell cycles in enriched-living mice. Theoretically, increased neurogenesis could deplete the population of progenitor cells in the subgranular zone, and experience-dependent demand for new neurons could compensate for this by shortening the cell cycle. The net result, that 4 weeks after BrdU injection significantly more BrdU-labeled neurons are present in enriched-living mice, is independent of these considerations. Future experiments will have to examine how cell cycle kinetics themselves are influenced by aging and by environmental stimulation.

Factors mediating the regulation of adult neurogenesis

Inheritable traits have different effects on three levels in the regulation of adult neurogenesis: proliferation, survival, and differentiation, suggesting the involvement of several regulatory genes (Kempermann et al., 1997a).

Neonatal handling of rats prevents age-related cell losses in CA1 and CA3 and deficits in spatial memory (Meaney et al., 1988). The hypothesized mechanism underlying this effect involves downregulation of glucocorticoid secretion. Glucocorticoids have also been shown to inhibit neurogenesis in the dentate gyrus (Gould et al., 1992). Glutamatergic afferents to the dentate gyrus also limit adult neurogenesis, as does psychosocial stress (Gould, 1994; Gould et al., 1997). In contrast, temporal lobe seizures caused an increase in neurogenesis in the dentate gyrus (Bengzon et al., 1997; Parent et al., 1997). Both results indicate a regulatory influence of glutamate on adult neurogenesis. In all of these cases effects on neurogenesis included strong stimulation of proliferation. Environmental stimulation is the first known modulator to interact with a later regulatory step of adult neurogenesis.

Mitogenic effects of growth factors have been described for progenitor cells from fetal and adult brain in vitro (Reynolds, 1992; Vescovi et al., 1993; Bartlett et al., 1994; Palmer et al., 1995; Ray et al., 1995). Epidermal growth factor, after intracerebroventricular infusion, shifted differentiation of BrdU-labeled cells in the granule cell layer to a glial fate (Kuhn et al., 1997).

\section{Does experience-dependent regulation imply a functional role?}

The extent to which spatial learning contributes to the behavioral improvements seen in our study remains undefined. However, even after living under standard conditions for 6 or 18 months, old mice stimulated by an enriched environment for as short as $68 \mathrm{~d}$ showed better performance in the water maze. This behavioral effect does not prove a causal link to the morphological results, but neurogenesis occurs in a context of functional effects.

Although the results might suggest that the new neurons are recruited for purposes of hippocampal function, further research is required to determine how and to what extent the neurons that are born during adulthood are integrated functionally into hippocampal circuitry. Anatomically, the new neurons extend neurites to CA3 as do other granule cells (Stanfield and Trice, 1988; Parent et al., 1997).

A functional interpretation of adult neurogenesis is supported by results from chickadees in which neurogenesis in the adult dentate gyrus has been shown to correlate with seasons in which the birds cover larger territories and have to remember numerous food storage sites (Barnea and Nottebohm, 1996).
The total numbers of neurons that can be generated in the senescent dentate gyrus, however, are low and it is important to determine whether these cells could contribute to the experiencerelated functional effects observed here. Various other hippocampal parameters have been shown to be inducible by living in an enriched environment (Jones and Smith, 1980). Some of these changes, such as plasticity of synaptic properties (Greenough et al., 1978), synaptic density (Saito et al., 1994), and dendritic arborization (Greenough and Volkmar, 1973), occur in older age and are likely to contribute to functional effects. However, the fact that the aging brain maintains a complex regulatory apparatus for the production of neurons in the dentate gyrus suggests that this plasticity has functional benefits. Our data support theories that place adult neurogenesis in a context of hippocampal function and shed new light on the potential for plasticity in the old brain.

\section{REFERENCES}

Altman J, Das GD (1964) Autoradiographic examination of the effects of enriched environment on the rate of glial multiplication in the adult rat brain. Nature 204:1161-1163.

Altman J, Das GD (1965) Autoradiographic and histologic evidence of postnatal neurogenesis in rats. J Comp Neurol 124:319-335.

Barnea A, Nottebohm F (1996) Recruitment and replacement of hippocampal neurons in young and adult chickadees: An addition to the theory of hippocampal learning. Proc Natl Acad Sci USA 93:714-718.

Bartlett PF, Kilpatrick TJ, Richards LJ, Talman PS, Murphy M (1994) Regulation of the early development of the nervous system by growth factors. Pharmacol Ther 64:371-393.

Bayer SA, Yackel JW, Puri PS (1982) Neurons in the rat dentate gyrus granular layer substantially increase during juvenile and adult life. Science 216:890-892.

Bengzon J, Kokaia Z, Elmér E, Nanobashvili A, Kokaia M, Lindvall O (1997) Apoptosis and proliferation of dentate gyrus neurons after single and intermittent limbic seizures. Proc Natl Acad Sci USA 94:10432-10437.

Bhatnagar M, Cintra A, Chadi G, Lindberg J, Oitzl M, De Kloet ER, Möller A, Agnatis LF, Fuxe K (1997) Neurochemical changes in the hippocampus of the brown Norway rat during aging. Neurobiol Aging 18:319-327.

Boyes BE, Kim SU, Lee V, Sung SC (1986) Immunohistochemical colocalization of $\mathrm{S}-100 \mathrm{~b}$ and the glial fibrillary acidic protein in rat brain. Neuroscience 17:857-865.

Cai L, Hayes NL, Nowakowski RS (1997) Local homogeneity of cell cycle length in developing mouse cortex. J Neurosci 17:2079-2087.

Cameron HA, Woolley CS, McEwen BS, Gould E (1993) Differentiation of newly born neurons and glia in the dentate gyrus of the adult rat. Neuroscience 56:337-344.

Caviness VS, Takahashi T, Nowakowski RS (1995) Numbers, time and neocortical neuronogenesis: a general developmental and evolutionary model. Trends Neurosci 18:379-383.

Coggeshall RE, Lekan HA (1996) Methods for determining numbers of cells and synapses: a case for more uniform standards for review. J Comp Neurol 364:6-15.

Gould E (1994) The effects of adrenal steroids and excitatory input on neuronal birth and survival. Ann NY Acad Sci 743:73-93.

Gould E, Cameron HA, Daniels DC, Woolley CS, McEwen BS (1992) Adrenal hormones suppress cell division in the adult rat dentate gyrus. J Neurosci 12:3642-3650.

Gould E, McEwen BS, Tanapat P, Galea LAM, Fuchs E (1997) Neurogenesis in the dentate gyrus of the adult tree shrew is regulated by psychosocial stress and NMDA receptor activation. J Neurosci 17:2492-2498.

Gower AJ, Lamberty Y (1993) The aged mouse as a model of cognitive decline with special emphasis on studies in NMRI mice. Behav Brain Res 57:163-173.

Greenough WT, Volkmar FR (1973) Pattern of dendritic branching in occipital cortex of rats reared in complex environments. Exp Neurol 40:491-504.

Greenough WT, West RW, DeVoogd TJ (1978) Postsynaptic plate perforations: changes with age and experience in the rat. Science 202:1096-1098. 
Guillery RW, Herrup K (1997) Quantification without pontification: choosing a method for counting objects in sectioned tissues. J Comp Neurol 386:2-7.

Gundersen HJ, Bagger P, Bendtsen TF, Evans SM, Korbo L, Marcussen N, Møller A, Nielsen K, Nyengaard JR, Pakkenberg B, Sørensen FB, Versterby A, West MJ (1988) The new stereological tools: disector, fractionator, nucleator and point sampled intercepts and their use in pathological research and diagnosis. Acta Pathol Microbiol Immunol Scand 96:857-881.

Jones DG, Smith BJ (1980) The hippocampus and its response to differential environments. Prog Neurobiol 15:19-69.

Kaplan MS, Bell DH (1984) Mitotic neuroblasts in the 9-day-old and 11-month-old rodent hippocampus. J Neurosci 4:1429-1441.

Kempermann G, Kuhn HG, Gage FH (1997a) Genetic influence on neurogenesis in the dentate gyrus of adult mice. Proc Natl Acad Sci USA 94:10409-10414.

Kempermann G, Kuhn HG, Gage FH (1997b) More hippocampal neurons in adult mice living in an enriched environment. Nature 386:493-495.

Kuhn HG, Dickinson-Anson H, Gage FH (1996) Neurogenesis in the dentate gyrus of the adult rat: age-related decrease of neuronal progenitor proliferation. J Neurosci 16:2027-2033.

Kuhn HG, Winkler J, Kempermann G, Thal LJ, Gage FH (1997) Epidermal growth factor and fibroblast growth factor-2 have different effects on neural progenitors in the adult rat brain. J Neurosci 17:5820-5829.

Kunstyr I, Leuenberger HGW (1975) Gerontological data on C57BL/6 mice. I. Sex differences in survival curves. J Gerontol 30:157-162.

Meaney MJ, Aitken DH, van Berkel C, Bhatnagar S, Sapolsky RM (1988) Effect of neonatal handling on age-related impairments associated with the hippocampus. Science 239:766-768.

Mullen RJ, Buck CR, Smith AM (1992) NeuN, a neuronal specific nuclear protein in vertebrates. Development 116:201-211.

Palmer TD, Ray J, Gage FH (1995) FGF-2-responsive neuronal progenitors reside in proliferative and quiescent regions of the adult rodent brain. Mol Cell Neurosci 6:474-486.
Palmer TD, Takahashi J, Gage FH (1997) The adult rat hippocampus contains premordial neural stem cells. Mol Cell Neurosci 8:389-404.

Parent JM, Yu TW, Leibowitz RT, Geschwind DH, Sloviter RS, Lowenstein DH (1997) Dentate granule cell neurogenesis is increased by seizures and contributes to aberrant network reorganization in the adult rat hippocampus. J Neurosci 17:3727-3738.

Ray J, Raymon HK, Gage FH (1995) Generation and culturing of precursor cells and neuroblasts from embryonic and adult central nervous system. Methods Enzymol 254:20-37.

Reynolds BA, Weiss S (1992) Generation of neurons and astrocytes from isolated cells of the adult mammalian central nervous system. Science 255:1707-1710.

Rosenzweig MR, Bennett EL (1996) Psychobiology of plasticity: effects of training and experience on brain and behavior. Behav Brain Res 78:57-65.

Rosenzweig MR, Krech D, Bennett EL, Diamond MC (1962) Effects of environmental complexity and training on brain chemistry and anatomy. J Comp Physiol Psychol 55:429-437.

Saito S, Kobayashi S, Ohashi Y, Igarashi M, Komiya Y, Ando S (1994) Decreased synaptic density in aged brains and its prevention by rearing under enriched environment as revealed by synaptophysin contents. J Neurosci Res 39:57-62.

Shepherd GM (1994) Neurobiology. New York: Oxford UP.

Silver LM (1995) Mouse genetics. New York: Oxford UP.

Stanfield BB, Trice JE (1988) Evidence that granule cells generated in the dentate gyrus of adult rats extend axonal projections. Exp Brain Res 72:399-406.

Takahashi T, Nowakowski RS, Caviness VS, Jr (1992) BUdR as an S-phase marker for quantitative studies of cytokinetic behaviour in the murine cerebral ventricular zone. J Neurocytol 21:185-197.

Vescovi AL, Reynolds BA, Fraser DD, Weiss S (1993) bFGF regulates the proliferative fate of unipotent (neuronal) and bipotent (neuronal/ astroglial) EGF-generated CNS progenitor cells. Neuron 11:951-966.

West MJ, Coleman PD, Flood DG, Troncoso JC (1994) Differences in the pattern of hippocampal neuronal loss in normal ageing and Alzheimer's disease. Lancet 344:769-772. 